\title{
Human herpesvirus DNA occurrence in intracranial aneurysmal wall: illustrative case
}

\author{
Nícollas Nunes Rabelo, MD, PhD, ${ }^{1}$ Antonio Carlos Samaia da Silva Coelho, ${ }^{1}$ João Paulo Mota Telles, ${ }^{1}$ Giselle Coelho, MD, PhD, ${ }^{1}$ \\ Caio Santos de Souza, ${ }^{2}$ Tania Regina Tozetto-Mendoza, $\mathrm{PhD},{ }^{2}$ Natan Ponzoni Galvani de Oliveira, ${ }^{2}$ \\ Paulo Henrique Braz-Silva, DDS, PhD, ${ }^{2,3}$ Manoel Jacobsen Teixeira, MD, PhD, ${ }^{1}$ and Eberval Gadelha Figueiredo, MD, $\mathrm{PhD}^{1}$
}

${ }^{1}$ Department of Neurosurgery, ${ }^{2}$ Laboratory of Virology (LIM-52), Institute of Tropical Medicine of São Paulo, School of Medicine, and ${ }^{3}$ Department of Stomatology, School of Dentistry, University of São Paulo, São Paulo, Brazil

BACKGROUND Subarachnoid hemorrhages secondary to intracranial aneurysms (IAs) are events of high mortality. These neurological vascular diseases arise from local and systemic inflammation that culminates in vessel wall changes. They may also have a possible relationship with chronic viral infections, such as human herpesvirus (HHV), and especially Epstein-Barr virus (EBV), which causes several medical conditions. This is the first description of the presence of HHV deoxyribonucleic acid (DNA) in a patient with IA.

OBSERVATIONS A 61-year-old woman with a downgraded level of consciousness underwent radiological examinations that identified a 10-mm ruptured aneurysm in the anterior communicating artery. A microsurgery clip was performed to definitively treat the aneurysm and occurred without surgical complications. Molecular analysis of the material obtained revealed the presence of EBV DNA in the aneurysm wall. The patient died 21 days after admission due to clinical complications and brain swelling.

LESSONS This is the first description of the presence of herpesvirus DNA in a patient with IA, presented in $2.8 \%$ of our data. These findings highlight that viral infection may contribute to the pathophysiology and is an additional risk factor for IA formation, progression, and rupture by modulating vessel wall inflammation and structural changes in chronic infections.

https://thejns.org/doi/abs/10.3171/CASE21301

KEYWORDS intracranial aneurysm; intracranial hemorrhage; subarachnoid hemorrhage; human herpesvirus; HHV; inflammation

Subarachnoid hemorrhage (HSA) from a ruptured intracranial aneurysm $(I A)$ is a leading cause of mortality worldwide among working-age patients. This clinical condition increases the risk of mortality by $50 \%$, but the mechanisms leading to structural vessel wall weakening are unclear. Some risk factors are known, including female gender, age under 50 years, smoking, hyperlipidemia, aneurysm size ratio, polycystic kidney, multiple aneurysms, old cerebral infarct, family history, systemic arterial hypertension, Marfan and collagen syndromes, and Japanese and Finnish ethnicity. ${ }^{1,2}$ Nevertheless, currently risk factors do not explain why some people develop IA disease, have worse disease progression, or even rupture. Some questions persist, so the main hypothesis of this study is that the presence of the virus in the brain wall might lead to more inflammatory influence in the artery.

Human herpesviruses (HHVs) are the cause of several clinical conditions. Many diseases, such as rhinitis, encephalopathies, hepatitis, esophagitis, colitis, pneumonia, neonatal infections, febrile vesicles, genital infections, vasculitis, ischemic attacks, thrombosis, atherosclerosis, cancer, neuroinflammatory diseases, giant cell arthritis, and granulomatous aortitis, have all been described as secondary to these viruses. ${ }^{2-6}$ Epstein-Barr virus (EBV), in particular, has been considered since 1990 to be an important pathogen in the pathophysiology of several

ABBREVIATIONS CMV = cytomegalovirus; DNA = deoxyribonucleic acid; EC = endothelial cell; EBV = Epstein-Barr virus; HC-FMUSP = São Paulo School of Medicine Clinics Hospital; HHV = human herpesvirus; HSV = herpes simplex virus; $\mathrm{IA}=$ intracranial aneurysm; $\mathrm{PCR}=$ polymerase chain reaction; $\mathrm{qPCR}=$ quantitative polymerase chain reaction; VZV = varicella zoster virus; WSS = wall shear stress. INCLUDE WHEN CITING Published August 23, 2021; DOI: 10.3171/CASE21301.

SUBMITTED May 15, 2021. ACCEPTED June 7, 2021.

(C) 2021 The authors, CC BY-NC-ND 4.0 (http://creativecommons.org/licenses/by-nc-nd/4.0/). 
diseases. ${ }^{7}$ EBV, in its latent form, is present in more than $90 \%$ of the adult population, with saliva as the primary route of transmission, and is detected mainly in $B$ lymphocytes and in the epithelium of oropharyngeal cells. $^{8,9}$

The recent worldwide concern about viral infection and neurological effects has led to the hypothesis of a relationship between chronic viral infection and cerebrovascular disease. The presence of chronic pathogens implies a local and systemic silent inflammatory response and plays a role in chronic vessel wall changes, leading a vasculitis and infestation viral in the brain wall. This report presents the first description of HHV deoxyribonucleic acid (DNA) encountered in an IA and discusses the possible contribution of viral infection to the pathophysiology of IA formation and rupture.

\section{Illustrative Cases}

The study selected 401 patients from the São Paulo School of Medicine Clinics Hospital (HC-FMUSP) Department of Neurological Surgery. The patients were divided into 2 groups: ruptured aneurysm (244) and unruptured aneurysm (177). We excluded (366) IA. It was possible to collect surgically and analyze just (35) IA samples; these comprised (22) ruptured aneurysm and (13) nonruptured aneurysm.

The protocol started with obtaining biological material through vascular microsurgery procedures to remove aneurysm fragment samples performed at the surgical center of the Central Institute Department of Neurosurgery at HC-FMUSP. After collection, all samples were sent to the Virology Laboratory of the Instituto de Medicina Tropical of São Paulo in a freezer at $-80^{\circ} \mathrm{C}$. The human pan herpesviruses (HSV-1, HSV-2, EBV, cytomegalovirus [CMV], varicella zoster virus [VZV], HHV-6, HHV-7, and HHV-8) were selected and analyzed by polymerase chain reaction (PCR) targeting. These viruses were chosen because of high frequency, the capacity to spread out, and for having a possible relationship with the pathophysiology of aneurysms.

The DNA extraction of genetic material was done manually using the Qiagen DNA extraction kit Minikit 250 (Qiagen). The process followed the instructions given by the manufacturer, which consist of obtaining purified DNA from a silica column. The extracted sample was subjected to PCR with primers for $\beta$-globin as an internal control of the reaction to confirm the effectiveness of DNA extraction through the process amplification. The sample was analyzed by spectrophotometry to check the amount of DNA extracted. This process was done using the Nanodrop 2000 spectrophotometer (Thermo Fischer).

For the identification of the 8 types of human herpesvirus in the aneurysm fragments, the PCR technique and the enzymatic digestion process were performed. Two PCR mixtures were prepared, each containing different primers, capable of identifying different herpesviruses. The primer pair VZV sense and antisense was used to identify HHV-6A, HHV-6B, HHV-7, and VZV, while the primer pair HSV was used to identify HSV-1, HSV-2, EBV, CMV, and HHV-8.

The mixtures were made in centrifuge tubes and contained 0.5 $\mu \mathrm{L}$ of primer sense and antisense already mixed, $5 \mathrm{U} / \mu \mathrm{L}$ TaqPolimerase, $2.5 \mu \mathrm{L}$ Gold Star $10 \times$ buffer, and nuclease-free water (Thermo Fischer). For the reaction, the samples were placed in a Proflex PCRsystem thermal cycler (Thermofisher). The reactions for the VZV and HSV primers have different cycles, so the samples were incubated in separate thermoblocks.
After the end of the cycle, a $1.5 \%$ agarose gel was made, mixing $1.5 \mathrm{~g}$ of HyAgarose (Hydragene), $100 \mathrm{~mL}$ of concentrated Tris-acetateEDTA (TAE) $1 \times$ (Invitrogen), and $0.8 \mu \mathrm{L}$ of ethidium bromide (Invitrogen). Samples with bands at heights of 500 and 600 base pairs were considered positive and separated for the enzymatic digestion process. The counting of the height of the base pairs was made by comparison with the molecular weight standard of 100 base pairs (Invitrogen). The enzymatic digestion process consists of making 2 mixtures, each mixture containing an enzyme. The restriction enzymes used for this pro-

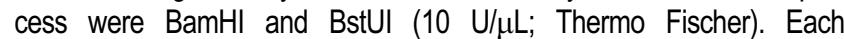
herpesvirus has a different band fragment under the action of these enzymes. The digestion mixtures were prepared with FastDigest Green Buffer digestion buffer (Thermo Fischer) restriction enzyme and distilled $\mathrm{H}_{2} \mathrm{O}$.

Biopsy samples were tested using the optimized quantitative PCR (qPCR) HHV-6 technique in-house. The qPCR was based on the Taqman probe system, using TAQMAN Universal Master Mix $2 \times$ concentrated from $5 \mu \mathrm{L}$ of the nucleic acid extraction product and a final volume of $25 \mu \mathrm{L}$.

qPCR was performed using QuantStudio 5 (Thermo Fischer Scientific) and analyzed using the device's own software. The qPCR cycling was determined from the buffer used.

This study analyzed 35 samples, and applying the protocol found 1 patient positive for HHV. A 61-year-old female smoker (200 pack-years) presenting with arterial hypertension sought medical attention due to a sudden and severe headache and a decreased level of consciousness. Her initial evaluation showed a blood pressure reading of $230 / 120 \mathrm{mmHg}$, a Glasgow coma scale score of 7 , a Hunt and Hess scale score of 3, a World Federation of Neurological Surgeons score of 5 , and photoreactive isochoric pupils upon orotracheal intubation. The patient was sedated (Ramsay scale 6) with photoreactive isochoric pupils and left hemiparesis.

A radiological examination revealed the presence of a $10-\mathrm{mm}$ ruptured aneurysm in the anterior communicating artery, with wall irregularities and saccular morphology, as well as symmetrical bilateral aneurysms (in mirror) of the middle cerebral arteries and moderate brain swelling, with an 8-mm midline deviation and a Fisher scale of 4 (Figs. 1 and 2).

The patient underwent microsurgery with a definitive clip, with no surgical complications. Nevertheless, the patient died on the 21 st day after admission from the clinical complications of vasospasm and brain swelling. An aneurysm sample was collected using sterile procedures after the vascular microsurgical clipping. The sample was preserved at $-80^{\circ} \mathrm{C}$ until PCR analysis.

Molecular analysis was performed in order to verify the possible presence of 1 of the 8 types of herpesviruses in the patient's aneurysmal wall. The procedure was conducted according to the technical specifications presented in Table 1. A positive PCR result was obtained for EBV (HHV-4), with a viral load of 12.163 copies $/ \mathrm{mL}$ (Fig. 3).

\section{Discussion \\ Observations}

EBV infections have been linked to the occurrence of some brain and vascular diseases. For example, the EBV latent protein EBNA1 and the early lytic protein BZLF1 were found in astrocytes and microglia, in addition to $B$ lymphocytes, in $82 \%$ of multiple sclerosis cases. The viral proteins were in an active state of transcription, indicating a possible correlation with the processes of inflammation and degeneration of neural tissue. A rare complication of EBV infection is encephalopathy, a 

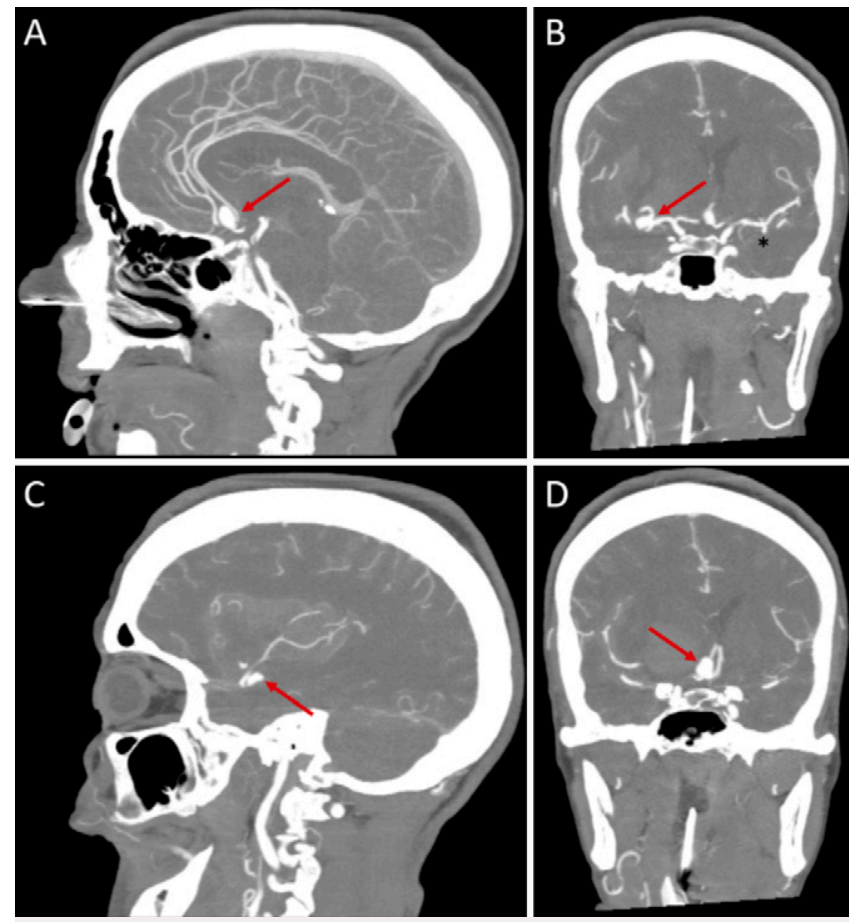

FIG. 1. Computed tomography (CT) angiography. A and B: CT angiography showing an aneurysm of the anterior communicating artery. The aneurysm was $10 \mathrm{~mm}$ in diameter, with a 2-mm neck and an irregular, saccular morphology, with angiographic vasospasm present, $40 \mathrm{~mL}$ in volume, indicated by red arrows. Asterisks in B indicate the middle cerebral arteries (MCA) in a mirror aneurysm. $C$ and $\mathrm{D}$ : CT angiography showing an aneurysm of the right MCA $6 \mathrm{~mm}$ in diameter, with a 3$\mathrm{mm}$ neck, and an irregular, saccular shape, with angiographic vasospasm present, $40 \mathrm{~mL}$ in volume, indicated by red arrows.

condition caused by the immunotoxicity of EBV infection. ${ }^{10}$ This complication is generally restricted to children and immunocompromised patients, but it can affect immunocompetent adult patients. ${ }^{10}$ Case reports also indicate the occurrence of acute transverse myelitis and a combination of polyradiculitis and anterior horn syndrome in immunocompetent patients, as well as the possible development of neurological sequelae. ${ }^{11}$

Chronic active EBV infections can show a wide variety of manifestations. The involvement of brain meninges, parenchyma, vascular system, epithelium, and lymphoid tissue has been reported as a cause of neurological disorders, such as dilation of spindle aneurysms, meningitis, meningoencephalitis, and disseminated encephalomyelitis. The vascular consequences on the central nervous system arise due to the direct invasion of the virus, the infiltration of lymphocytes infected by EBV, and the deposition of antigen-antibody complexes in the endothelium. ${ }^{12}$

One of the major causes of morbidity in cardiovascular and cerebrovascular diseases is atherosclerosis. The etiopathogenesis of atheromatous plaques is similar to the chronic inflammatory process, and establishing an association with viral infections is possible. Studies have shown a high prevalence of EBV, as well as CMV and Torque Teno Vírus (TTV), in atheroma plaques, supporting the hypothesis of viral etiology, especially in patients with vascular disorders. ${ }^{13}$

High wall shear stress (WSS) can trigger proinflammatory signaling in endothelial cells (ECs), which then recruit macrophages through expression of chemoattractant protein 1 (MCP1). This process leads to the expression of proteases that are responsible for disruption of the vessel's elastic lamina and for collagen remodeling, which includes changes in the orientation and type of fibers. These changes, in turn, lead to a focal outward bulging of the wall and the formation of intracranial aneurysms. In addition, high WSS leads to dysfunction and disruption of the ECs and generates an inflammatory process, which implies migration, changes in phenotype, and apoptosis of vascular smooth muscle cells through biochemical reactions. ${ }^{14}$

Inflammation therefore determines the aneurysm formation and its progression to rupture. Consequently, modulating this process may be clinically significant. ${ }^{15}$ There are some studies that established CMV in abdominal aortic aneurysm, reinforcing the idea of virus influence in vascular wall response. ${ }^{6}$
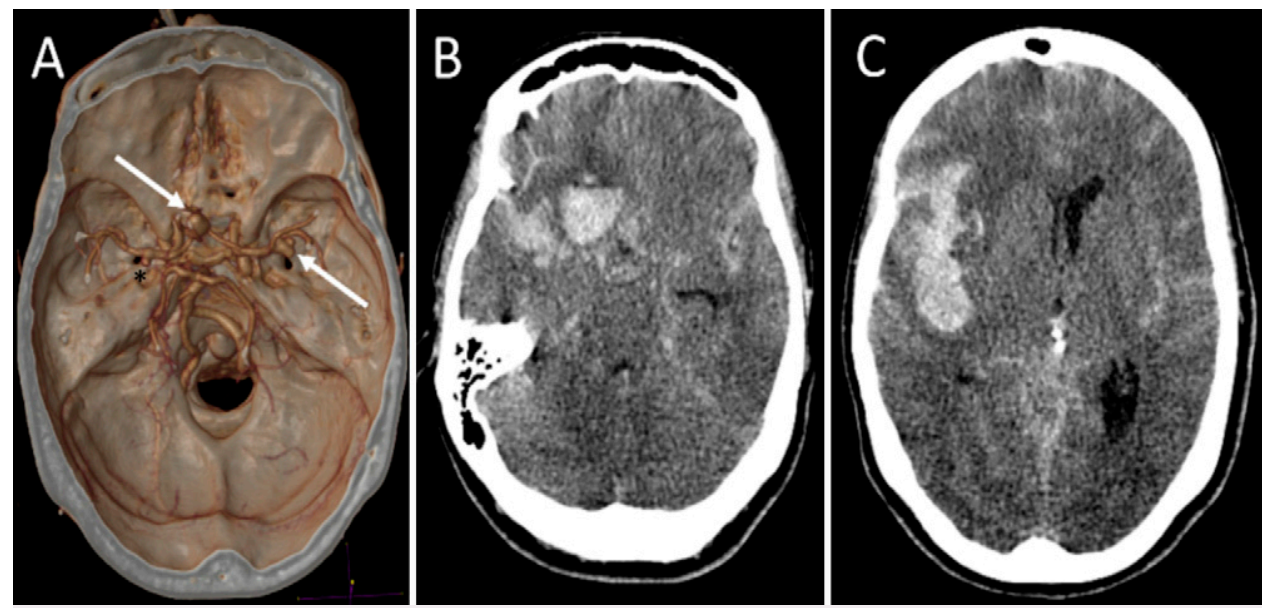

FIG. 2. Computed tomography (CT) angiography reconstruction and CT scan in axial view. A: Computerized reconstruction showing the anterior cerebral circulation with aneurysms in the anterior communicating artery, right middle cerebral artery (MCA). The red arrow indicates the MCA, and the asterisk represents a mirror MCA aneurysm. B and C: CT scans showing moderate brain swelling and bleeding, with an 8-mm deviation from the midline (modified Fisher and Fisher scale 4). 
TABLE 1. Technical specification of the virology analysis

\begin{tabular}{ccccccccc}
\hline Nucleic Acid & A260 (abs) & A280 (abs) & $260 / 280$ & $260 / 230$ & Sample Type & Internal Control & Template Control & Viral Load \\
\hline $26 \mathrm{ng} / \mu \mathrm{L}$ & 52 & 41 & 127 & 34 & DNA & $\beta$-globulin & 36.315 & 12.163 \\
\hline
\end{tabular}

$\mathrm{A} 260=$ ratios of the absorbance value of $260 \mathrm{~nm} ; \mathrm{A} 280=$ ratios of the absorbance value of $280 \mathrm{~nm}$; abs = antibodies.

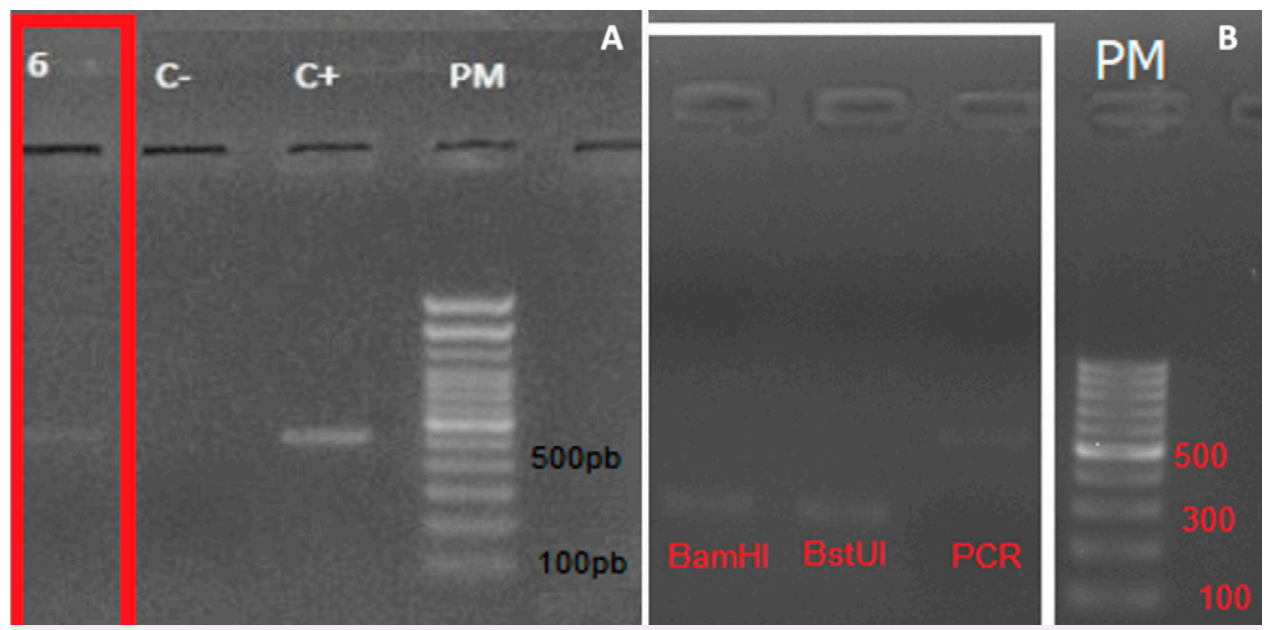

FIG. 3. Sample of the wall of the intracranial aneurysm showing human herpesvirus. A: Five hundred-base pair fragment visualized on a $1.5 \%$ gel. The pattern corresponds to herpesvirus. $\mathrm{C}+=$ positive control; $\mathrm{C}-\mathbf{=}$ negative control; $\mathrm{PM}=$ molecular weight. $\mathrm{B}$ : EBV band fragment pattern after enzymatic digestion. The first fragment was generated by the BamHI enzyme, the second by BstUI, and the third is the product of PCR amplification. $\mathrm{pb}=$ base pair.

Chronic local inflammation might lead to artery systemic inflammation, weaken the arterial wall, and, consequently, form an $\mathrm{Al}$, as well as assist with its rupture. This salient chronic infection can add to tradition risk factors (smoking, hypertension, and history family) and lead to aneurysm formation and rupture.

There are several limitations of this study. First, the interpretation of the results is limited by the small size of the sample, which is supported because of the technical difficulty to have eligible patient to have the sample collected in operating room. However, this is the first report to analyze HHV in a brain sample. More studies are needed to determine the exact relationship and establish cytokines and biomarkers involved in IA. Nevertheless, these findings may highlight a unique and important discussion regarding the understandable concern over the development of IA and rupture.

\section{Lessons}

This is the first description of the presence of herpesvirus DNA in a patient with $I A$, presented in $2.8 \%$ of our data. These findings highlight that viral infection may contribute to the pathophysiology and risk factors of IA formation, progression, and rupture by modulating vessel wall inflammation. More studies are needed to determine the exact relationship and to establish the cytokines and biomarkers involved in the viral IA infection.

\section{Acknowledgments}

We thank Professors Eberval Gadelha Figueiredo and Manoel Jacobsen Teixeira for their attention and notable dedication in the development of this paper and for support of FMUSP, as well as
Marcia Nunes Rabelo, Roberto Costa Rabelo, and Neiffer Nunes Rabelo for their strong and continuous support.

\section{References}

1. Pyysalo MJ, Pyysalo LM, Pessi T, Karhunen PJ, Öhman JE. The connection between ruptured cerebral aneurysms and odontogenic bacteria. J Neurol Neurosurg Psychiatry. 2013;84(11): 1214-1218.

2. Rabelo NN, Rodrigues RS, Massoud Salame AA, Braz-Silva PH, Teixeira MJ, Figueiredo EG. Letter to the Editor. Do bacteria contribute to formation and rupture of intracranial aneurysms? J Neurosurg. 2019;132(6):2016-2017.

3. Sezgin E, An P, Winkler CA. Host genetics of cytomegalovirus pathogenesis. Front Genet. 2019;10:616.

4. Hajjar DP, Fabricant CG, Minick CR, Fabricant J. Virus-induced atherosclerosis. Herpesvirus infection alters aortic cholesterol metabolism and accumulation. Am J Pathol. 1986;122(1):62-70.

5. Kempe S, Heinz P, Kokai E, Devergne O, Marx N, Wirth T. EpsteinBarr virus-induced gene-3 is expressed in human atheroma plaques. Am J Pathol. 2009;175(1):440-447.

6. Gredmark-Russ S, Dzabic M, Rahbar A, et al. Active cytomegalovirus infection in aortic smooth muscle cells from patients with abdominal aortic aneurysm. J Mol Med (Berl). 2009;87(4): 347-356.

7. Haffajee AD, Socransky SS. Microbial etiological agents of destructive periodontal diseases. Periodontol 2000. 1994;5:78-111.

8. Slots J, Sabeti M, Simon JH. Herpesviruses in periapical pathosis: an etiopathogenic relationship? Oral Surg Oral Med Oral Pathol Oral Radiol Endod. 2003;96(3):327-331.

9. Cappuyns I, Gugerli P, Mombelli A. Viruses in periodontal disease a review. Oral Dis. 2005;11(4):219-229. 
10. Poorthuis MHF, Battjes S, Dorigo-Zetsma JW, de Kruijk JR. Primary Epstein-Barr virus infection in immunocompetent patients with acute transverse myelitis and a combination of polyradiculitis and anterior horn syndrome as neurological manifestations. BMJ Case Rep. 2018;2018:bcr2018225333.

11. Johnson G, Nelson S, Petric M, Tellier R. Comprehensive PCRbased assay for detection and species identification of human herpesviruses. J Clin Microbiol. 2000;38(9):3274-3279.

12. Hassani A, Corboy JR, Al-Salam S, Khan G. Epstein-Barr virus is present in the brain of most cases of multiple sclerosis and may engage more than just B cells. PLoS One. 2018;13(2):e0192109.

13. Priyanka S, Kaarthikeyan G, Nadathur JD, Mohanraj A, Kavarthapu A. Detection of cytomegalovirus, Epstein-Barr virus, and Torque Teno virus in subgingival and atheromatous plaques of cardiac patients with chronic periodontitis. J Indian Soc Periodontol. 2017;21(6):456-460.

14. Frösen J, Cebral J, Robertson AM, Aoki T. Flow-induced, inflammation-mediated arterial wall remodeling in the formation and progression of intracranial aneurysms. Neurosurg Focus. 2019;47(1):E21.

15. Rabelo NN, Teixeira MJ, Figueiredo EG. Letter by Rabelo et al regarding article "potential influences of gut microbiota on the formation of intracranial aneurysm". Hypertension. 2019 Jul;74(1):e1. Published online May 6, 2019. doi: 10.1161/HYPERTENSIONAHA.119.12829.

\section{Disclosures}

The authors report no conflict of interest concerning the materials or methods used in this study or the findings specified in this paper.

\section{Author Contributions}

Conception and design: Rabelo, Samaia da Silva Coelho, Tozetto Mendoza, Braz-Silva, Figueiredo. Acquisition of data: Rabelo, Telles, de Souza, Tozetto Mendoza, Braz-Silva, Figueiredo. Analysis and interpretation of data: Rabelo, Samaia da Silva Coelho, Telles, Tozetto Mendoza, Galvani de Oliveira, Braz-Silva, Figueiredo. Drafting the article: Rabelo, Samaia da Silva Coelho, Telles, Tozetto Mendoza, Galvani de Oliveira, Figueiredo. Critically revising the article: Rabelo, Samaia da Silva Coelho, Coelho, Galvani de Oliveira, Braz-Silva, Teixeira, Figueiredo. Reviewed submitted version of manuscript: Rabelo, Samaia da Silva Coelho, Coelho, de Souza, Galvani de Oliveira, Braz-Silva, Figueiredo. Approved the final version of the manuscript on behalf of all authors: Rabelo. Statistical analysis: Telles. Administrative/technical/material support: Coelho, Braz-Silva, Figueiredo. Study supervision: Coelho, Figueiredo.

\section{Correspondence}

Nícollas Nunes Rabelo: School of Medicine, University of São Paulo (FMUSP), São Paulo, Brazil. nicollasrabelo@hotmail.com. 